\title{
LA BÚSQUEDA DEL SENTIDO DE VIDA EN RELACIÓN CON LOS ESPACIOS EN LA OBRA LOS HOMBRES INVISIBLES de Mario Mendoza*
}

\author{
THE SEARCH FOR THE MEANING OF LIFE IN \\ RELATION TO THE SPACES IN THE WORK LOS \\ HOMBRES INVISIBLES BY MARIO MENDOZA
}

Natalia Andrea Cardona Jaramillo ${ }^{1}$

Artículo derivado de la investigación
Estudios de literatura colombiana, Grupo de
Investigación Humanitas de la Universidad
Católica de Oriente.

Cómo citar este artículo: Cardona Jaramillo, N. A. (2019). La búsqueda del sentido de vida en relación con los espacios en la obra Los hombres invisibles de Mario Mendoza. Estudios de Literatura Colombiana 44, pp. 151-164. DOI: https://doi.org/10.17533/ udea.elc.n44a09

naty4857@yahoo.es

Universidad Católica de Oriente, Colombia

Recibido: 01.05.2018

Aprobado: 01.10.2018
Copyright: $₫ 2019$ EstudiosdeLiteratura Colombiana. Este es un artículo de acceso abierto distribuido bajo los términos de la Licencia Creative Commons AtribuciónNo comercial - Compartir igual 4.0 Internacional
Resumen: el presente texto tiene como propósito analizar la búsqueda de 'sentido de vida' en relación con los espacios en la novela Los hombres invisibles del escritor Mario Mendoza. Para ello se toma como referencia dicho concepto a partir de los presupuestos de Viktor Frankl y Alfred Längle en la Logoterapia y el Análisis Existencial, respectivamente, a través de los cuales se logra demostrar que encontrar el sentido de vida es un imperativo en el ser humano, y que en dicha consecución son determinantes los espacios en los cuales este se establece y desarrolla su proyecto de vida.

Palabras clave: sentido de vida; novela colombiana; espacio urbano; selva; psicoterapia.

\begin{abstract}
The following text has as purpose analyze the research for the meaning of life in relation with the spaces in the novel Los hombres invisibles, by the writer Mario Mendoza. For that it is taken as a reference the concept meaning of life from the Viktor Frankl's and expositions and Alfred Längle in the Logotherapy and Existential Analysis, through which it is possible to demonstrate that finding the meaning of life is an imperative in the human being, and that sense, is determinant the spaces in which he sets and develops his life's project.
\end{abstract}

Keywords: sense of life; Colombian novel; urban space; jungle; psychotherapy. 


\section{Introducción}

Mario Mendoza, nacido en Bogotá en el año 1964, es uno de los escritores colombianos actuales cuya producción literaria ha estado enfocada en la novela y recientemente en la novela juvenil. Inicia su recorrido como escritor con el libro de cuentos La travesía del vidente, el cual le hizo merecedor del Premio Nacional de Literatura del Instituto Distrital de Cultura y Turismo de Bogotá en el año 1995. En 1997 publica La ciudad de los umbrales, que es propiamente su primera novela; desde entonces, el autor presenta un amplio proceso de escritura sobre la ciudad de Bogotá, ${ }^{1}$ la cual ha querido retratar desde una perspectiva crítica, pues en su obra el espacio urbano va a entorpecer progresivamente las dinámicas de los seres que la habitan.

La novela Los hombres invisibles, publicada en el año 2007, tiene como protagonista a Gerardo Montenegro, un hombre que vive en un periodo determinado de su vida una serie de eventos que lo llevan a replantear su existencia, a cuestionarse las diferentes relaciones con los seres que ama y con el mundo en general. En un tiempo breve ocurre la muerte de sus padres, y se divorcia. Su trabajo como actor se estanca y su vida se convierte en un desastre.

La pregunta por el sentido de la vida que se plantea este personaje nace al contemplar que todo aquello en lo cual basaba su seguridad en el mundo se ve alterado, y ello lo mueve a realizar cambios importantes. Es así como en el protagonista surge la necesidad de huida, y la manera de materializarla es emprender un viaje en el cual desaparece la persona que había sido, con sus rutinas, su empleo y sus seres queridos. Esto lo lleva inevitablemente a cuestionarse y a descubrir quién es realmente él, o quién quiere ser, al margen de lo que hasta ese momento constituía a Gerardo Montenegro.

El texto que a continuación se presenta abordará la noción o categoría de sentido de vida de acuerdo con la propuesta de La Logoterapia

1 El proyecto de escritura sobre la ciudad de Bogotá lo comprenden las novelas: Scorpio City (1998), Relato de un asesino (2001), Satanás (2002) —novela con la cual recibe el Premio Biblioteca Breve de Seix Barral y es llevada al cine por Andrés Baiz-, Cobro de sangre (2004), Los hombres invisibles (2007), Buda Blues (2009), Apocalipsis (2011) y Lady Masacre (2013). Posterior a este periodo, Mario Mendoza continúa con un tipo de escritura alternativo con obras como Paranormal Colombia (2014), y en 2015 inicia la primera entrega de seis libros que componen la saga juvenil; El mensajero de Agartha (2015), La melancolía de los feos (2016), El libro de las revelaciones (2017) y su más reciente publicación, El diario del fin del mundo (2018). 
de Viktor Frankl y el Análisis Existencial desde la perspectiva de Alfred Längle; seguidamente, se identifica el modo en que opera esta categoría en un momento específico de la novela mencionada y el papel que en ello desempeñan los distintos espacios, específicamente el contraste entre la ciudad y la jungla.

\section{La pregunta por el sentido de vida}

Si bien el ser humano se ha planteado la pregunta por la existencia a lo largo de la historia, la necesidad de responder a tal cuestionamiento aumenta cuando las dificultades o momentos coyunturales de la vida se presentan con toda su fuerza. El sentimiento de incertidumbre crece y hace que se emprenda la búsqueda por el sentido de vida, y este se convierta en un imperativo.

En este marco surge el concepto de sentido de vida, el cual es dilucidado por el psiquiatra y neurólogo vienés Viktor Frankl, a través de la corriente psicológica que denomina Logoterapia. Aunque Frankl comparte dicho concepto de sentido de vida con su maestro Alfred Adler, toma distancia de este y de su propuesta de Psicología individual, que concibe al ser humano como un individuo indivisible que se constituye en relación con los demás y alcanza su realización personal al superar el sentimiento de inferioridad, y de esta manera llega a ser parte del colectivo, lo que configura Adler como "sentimiento comunitario" (Chávez, 2009, p. 37). Así, el enfoque sobre el sentido de vida de la psicología individual de Adler se centra en un yo social; Frankl, por su parte, hace énfasis en una psicoterapia que busca el sentido de vida a través de la autorreflexión y autotrascendencia, un yo existencial y espiritual.

Es así como Frankl, si bien reconoce el aporte de la psicología individual, escuela a la cual pertenecía, señala que dicha corriente aborda el sentido de vida desde una visión más vitalista y deja de lado la dimensión espiritual o humana, en la que él posteriormente concentró su trabajo:

Según Freire (2002), la teoría de la logoterapia se construye a partir de argumentaciones discrepantes a la perspectiva psicologista de Adler y, principalmente, a los presupuestos de Freud. En realidad no se trata de la oposición que descarta o que niega a la otra propuesta, sino una oposición a las limitaciones de aquellas dos visiones reduccionistas del ser humano (Chávez, 2009, p. 37).

De esta manera, más que reemplazar la psicoterapia de Adler o el psicoanálisis de Freud, Frankl pretende desarrollar un cuerpo teórico complementario que sepa ubicar la dimensión espiritual o del sentido de vida. 
La logoterapia es pues definida como "terapia del sentido" o "psicoterapia centrada en el sentido" (Frankl, 1967), y su autor la valida desde su propia experiencia como prisionero en los campos de concentración nazi, durante la Segunda Guerra Mundial. Posteriormente, su teoría de logoterapia fue complementada con el Análisis Existencial.

Para el abordaje del presente artículo, tomamos el concepto sentido de vida planteado por Viktor Frankl en su obra El hombre en busca de sentido (1991), en donde lo describe en los siguientes términos:

La búsqueda por parte del hombre del sentido de la vida constituye una fuerza primaria y no una "racionalización secundaria" de sus impulsos instintivos. Este sentido es único y específico en cuanto es uno mismo y uno solo quien tiene que encontrarlo; únicamente así logra alcanzar el hombre un significado que satisfaga su propia voluntad de sentido (p. 57).

A partir de la definición aportada por Frankl, se aborda ahora la novela de Mendoza, en la que el protagonista, Gerardo Montenegro, vivencia esa fuerza primaria como la necesidad de encontrarle significado a su existencia, y el camino que emprende en solitario para dicha búsqueda ratifica el hecho de que solo él puede encontrarlo. ${ }^{2}$

El sentido de vida que se pretende analizar en el protagonista está en relación directa con la ciudad y con lo que ello representa para la realización de la propia voluntad de sentido. Como bien lo dice Frankl (1991), el sentido de vida es una búsqueda individual, en la cual el significado que le asigna a su existencia es único y orientado por la voluntad del individuo.

Justamente, la decisión que toma Gerardo respecto a la reorientación que requería su vida surge de una reflexión tras un momento determinante; luego de pasar por la clínica de reposo en la cual fue internado por alcoholismo, consecuencia de lo que significó para él el abandono de su esposa, se muestran la voluntad individual y la decisión en Gerardo para recomponer su vida:

Ya me aburrí de estar aquí metido. Necesito salir y trabajar, conseguir otro apartamento y comprar algunas cosas [...] En efecto, a partir de ese día empecé a reconstruir mi vida y a acostumbrarme a que ahora estaba solo y que era preciso modificar mis hábitos y mis rutinas más elementales (Mendoza, 2007, p. 31).

$\overline{2}$ La novela inicia con una serie de eventos dolorosos en la vida de Gerardo Montenegro, (la muerte de sus padres y el abandono de su esposa), que lo lleva a empezar de cero. Para ello decide salir de Bogotá e iniciar un viaje en busca de "los hombres invisibles", una comunidad indígena de la cual había tenido datos por un paciente psiquiátrico y antropólogo, quien le insistía en que huyera de la ciudad y fuera tras su rastro. Esta invitación se le presenta como una revelación. Al ir tras las huellas de esta comunidad, Gerardo termina estableciéndose en la selva chocoana y descubre que el sentido de su vida yace en el encuentro fraterno con otros, en una vida sencilla y de servicio. 
El ejercicio de introspección de Gerardo inicia con reconocerse como un individuo a cargo de sí mismo; su soledad le permitió identificar los dos elementos fundamentales que requiere: libertad y responsabilidad para asumirse en la nueva dinámica de vida que decida planificar y llevar a cabo.

Al respecto, señala Frankl:

El ser humano al tomar consciencia de su estar en el mundo, descubrirá que tiene la tarea de hacerse cargo, en primer lugar, de su propia vida. Este descubrimiento puede manifestarse en la consciencia humana de modo ambivalente. Para algunas personas puede significar el gozo de emprender el proyecto de definición de la propia existencia y para otras significará la angustia de sentirse arrojadas en el mundo bajo el peso de una vida que ellas no eligieron (citado por Pareja Herrera, 1998, pp. 146-147).

La responsabilidad y la libertad son partes constitutivas de la construcción del sentido de vida. Por ello, aceptar la responsabilidad que se tiene sobre la propia vida y responder a los retos que plantea es un ejercicio de libertad, que a la vez se convierte en responsabilidad en la medida en que las elecciones generan compromiso con respecto a las consecuencias de cada acto. Por lo tanto, la verdadera libertad radica en la capacidad de elegir.

En relación con lo expuesto por Frankl, en la novela Los hombres invisibles se puede observar que su protagonista cavila ante la decisión de salir de la ciudad: "Cada quien es responsable de su vida y la termina de acuerdo con lo que elige. Ese no era mi problema, y no lo era porque mi vida tenía ahora un destino estrictamente individual" (Mendoza, 2007, p. 250). La responsabilidad sobre su futuro y sus elecciones le dan la contundencia y el respaldo a sus palabras, y a partir de allí forja su sentido de vida.

Ahora bien, además de lo expuesto por Viktor Frankl en la logoterapia respecto al sentido de vida, el Análisis Existencial afirma, según Alfred Längle, que es la libertad y la responsabilidad lo que fundamenta la toma de decisiones, y amplía el panorama al proponer que la pregunta por la existencia no solo es individual, pues también depende particularmente del propio contexto.

Desde una perspectiva existencial, la realización de la existencia humana se caracteriza por la toma de decisiones (libertad, responsabilidad). Mientras que la existencia de un individuo es experimentada únicamente de mi pertenencia, el proceso de llegar a una decisión está muy influenciado por el contexto del mundo particular de esa persona (Längle, 2007, p. 8).

La influencia del contexto, como bien lo sostiene Längle, cobra suma importancia en la toma de decisiones, lo cual queda demostrado en la novela 
cuando Gerardo hace un balance de su vida y percibe su realidad lamentable: ninguno de sus seres queridos está a su lado, no tiene un empleo y tanto económica como emocionalmente se encuentra devastado. Este contexto, indudablemente, contribuye en su decisión respecto a hacerse cargo de su existencia.

Ahora bien, aunque el Análisis Existencial fue propuesto inicialmente por Frankl, es a través de los planteamientos de Alfred Längle que esta se consolida. Dicha teoría puede considerarse como una corriente independiente que representa una visión antropológica, la cual ha evolucionado desde una "Logoterapia que complementa a las diversas psicoterapias" (Längle, 2007, p. 29) hasta un método psicoterapéutico completo, el cual busca sensibilizar al sujeto y ayudarlo en la realización personal pese a las exigencias y dificultades que se le puedan presentar en su entorno:

Para el Análisis Existencial la estructura de la existencia está compuesta por cuatro dimensiones y cada a una de ellas se corresponde con lo que llamamos motivaciones fundamentales del ser humano. De acuerdo a la psicoterapia existencial, la existencia humana está motivada por mucho más que los impulsos sexuales, la voluntad de poder, equilibrium o la liberación de la tensión (Längle, 2007, p. 30).

Tales dimensiones o categorías fundamentales del Análisis Existencial se presentan desde el individuo y en relación con otros aspectos. La primera es la relación con el mundo; la segunda, con la vida; la tercera, consigo mismo, y la cuarta, con el futuro. Cada una de ellas se corresponde con lo que Längle (2007) llama "motivaciones fundamentales del ser humano", que se presentan como: poder ser, tener gusto por vivir, ser como se es, y deber actuar o desarrollar lo valioso (p. 14).

Es a partir de esta perspectiva teórica expuesta por Frankl y Längle que cobra importancia el modo en que Gerardo Montenegro se da a la tarea de otorgarle un sentido a la existencia y la manera como esto lo vivencia en la obra, al ir tras la pista de las respuestas que tanto busca.

A continuación se presentan las motivaciones propuestas a partir del Análisis Existencial y su correspondencia con las etapas del proceso que atraviesa Gerardo Montenegro en la búsqueda de sentido de vida. Cabe resaltar que dicha teoría despliega la motivación junto a una o varias preguntas categóricas que reafirman o explican cada una de ellas.

La primera motivación permite cuestionar lo que una persona quiere y puede ser, para lo cual se parte del reconocimiento de la propia existencia al decir yo soy, y a continuación definir con qué herramientas se cuenta para el puedo ser, tal como se muestra a continuación: 
Soy - ¿Puedo ser? Tal pregunta toma en consideración tanto las circunstancias concretas (hechos) de mi ser aquí en primer lugar, así como también mi propio poder para soportar o cambiar las actuales condiciones de mi vida. Para lograrlo necesitamos tres requisitos previos importantes: espacio, protección y apoyo (Längle, 2007, pp. 10-11).

Llegado el momento de hacer un balance de su propia vida, Gerardo debe reconocerse como un hombre que en sus condiciones actuales no tiene idea de quién quiere ser realmente, y en vista de ello, como si se tratara de un nuevo proyecto, resuelve olvidarse de la persona que ha sido hasta entonces. Sin embargo, su forma de pensar no tiene correspondencia con su modo de vida, por lo que considera que el marcharse haica otro lugar le ayudará en esa intención de replantearse y decidir quién puede ser. Dado que Gerardo privilegia el espacio entre los elementos importantes para llevar a cabo el poder ser, decide cambiar su lugar y alejarse de la ciudad; en contraste, se propone la selva, lo que indica evidentemente un cambio drástico en relación con el espacio y con todo lo que desea reformar.

En la medida en que crece la desazón de Gerardo Montenegro por las diversas situaciones vividas, la ciudad se le presenta progresivamente como un lugar hostil, gris y convulsionado. Es así como al emprender la búsqueda de sí mismo, el espacio se convierte en el punto de partida. Por ello, alejarse de la ciudad y de lo que esta representaba era la búsqueda de la paz y la armonía que le podría brindar un espacio diferente y de esta manera pensarse como un hombre nuevo. En este sentido, la selva es considerada por Gerardo como el espacio ideal, ya que al representar lo opuesto a las urbes, le generaba mayor seguridad para su objetivo: dejar de ser quien era en la ciudad, para poder ser en la selva.

De igual modo, la confianza en sí mismo y en el mundo se construye con otros componentes importantes en esta primera motivación. La protección y el sostén, representados en algo o alguien, así como la experiencia, fundamentan la confianza en el ser, es decir, su estancia en el mundo. Como se ha dicho, Gerardo no contaba con el respaldo ni la compañía de sus seres queridos, y su experiencia de vida más cercana estaba marcada por eventos negativos, lo cual aceleró el proceso en cuanto a la primera motivación: ratificarse en el mundo y poder ser. Lo anterior puede observarse en el siguiente relato:

La ausencia de un afecto real que me retuviera, me confirmó que la vida de Gerardo Montenegro estaba liquidada, que lo mejor era dejarla atrás e iniciar otra en un paraje que no tuviera nada que ver con el que había marcado al funesto personaje (Mendoza, 2007, p. 164). 
Consolidar la protección y el sostén que requiere su vida es una necesidad que Gerardo evalúa, y por ello decide poder ser y estar en el mundo, determinando las condiciones de ese nuevo espacio, el cual debía estar despojado de todo lo que lo identificara con el antiguo yo. Ser y estar para él son dos acciones que indican un cambio trascendente en su vida: será una nueva persona y estará en otro lugar.

Ahora bien, la motivación de ser y estar en el mundo también requiere de dos elementos fundamentales: el poder soportar y aceptar. Tal como afirma Längle (2009), en ellos "crea el ser humano su espacio, el que necesita para su Dasein; lo que yo puedo aceptar, lo que puedo soportar, me deja espacio para ser" (p. 16). Aceptar lo difícil y soportar lo que no se puede cambiar se convierten en las primeras certezas y, a su vez, en la fuerza de transformación necesaria para Gerardo; de allí que encontrar su lugar en el mundo sea algo que adquiere un sentido físico y espiritual.

La segunda motivación está en relación con el continuo desarrollo y los cambios en la vida, y se refiere a tener ganas de vivir. Esta motivación invita a evaluar el deseo de vivir, y por ello pregunta: "Estoy aquí - ¿Es bueno?", y con esta, expone otras preguntas escrutadoras que confirman la segunda motivación:

Estoy aquí por un tiempo — ¿Con qué fin? ¿Con qué propósito? ¿Con cuáles habilidades o en qué contextos más grandes me veo a mí mismo? ¿Para qué vivo? - Esta dimensión de la existencia torna a nuestras contribuciones a la vida en un todo con sentido. Una persona experimenta el sentido cuando siente que se ha comprometido en tareas dignas o que se encuentra orientada a posibilidades que se develarán en el futuro (Längle, 2007, p. 12).

En la novela, con relación al continuo desarrollo y cambio, Gerardo considera preciso hacer una revisión sobre el hecho de vivir e identificar su sentido. En esta segunda motivación se hace consciente del valor de la vida en sí y la necesidad de hacerse a cargo de la decisión de vivir como es su deseo y con todo lo que esto implica. Al respecto afirma:

No basta con vivir, es preciso verse vivir y corregir cualquier movimiento en falso que realicemos. Si el rol no está escrito por nosotros mismos y no nos satisface, la solución es simple: paramos la representación, le agradecemos al público su presencia, mandamos al director al quinto infierno y nos salimos a la calle a aguantar hambre si es el caso. Porque es preferible un final trágico a uno mediocre, baboso y sin carácter (Mendoza, 2007, p. 13).

Gerardo asume su existencia y decisiones más allá de los convencionalismos sociales, y demuestra sus ganas de vivir al ratificar que está más allá de la aprobación de los demás; para ello lo somete todo a su 
propio juicio y ajustes. La segunda motivación se cumple en Gerardo, al definir para sí qué es digno en las elecciones de vida que hace y que están en concordancia con su deseo.

Con respecto a llevar a cabo esta segunda motivación, Längle añade: "Para apoderarme de mi vida y amarla, se necesitan tres cosas: relación, tiempo y cercanía" (Längle, 1998, p. 4). El relacionarse con otros y establecer cercanía pone de plano un aspecto fundamental, el de tener la posibilidad de sentirse tocado; es decir, de tener la experiencia de los demás en la vida propia, y con ello buscar la armonía con el mundo y consigo mismo.

El tiempo, de igual manera, adquiere su importancia en tanto es la inversión que se hace en la consolidación de las relaciones con los demás; en palabras de Längle (1998), "Tomarse un tiempo significa regalar una parte de la vida" (p. 4), es decir, dar de su propia vida a la de los demás, y en esa medida establecer lazos con otros; esto se logra con la cantidad de tiempo que se permanezca en la compañía de los demás, y así reafirmar el significado de la existencia, tener ganas de vivir.

En su travesía, Gerardo experimenta dichos componentes de cercanía, relación y tiempo, y es a través de ellos que recupera el sentido, el deseo de vivir. Las primeras personas que encuentra tras dejar la ciudad, lleno de soledad y amargura, es Mariano, un profesor y sindicalista quien se convierte en su amigo, y Candela; esa relación se consolida con el tiempo que comparten, así como los ideales y motivaciones que posteriormente asume como suyos.

Mi amistad con Mariano y con Candela se fortaleció con el paso de los días. Me di cuenta que la buena fortuna me los había puesto en el camino y disfrutaba de veras de la empatía que cada vez nos unía más a los tres [...]. Y con gran naturalidad, sin forzar la situación, me habían aceptado como el tercer integrante de una confraternidad divertida, pues yo no dejaba de reírme de ese par de viejos que se la pasaban maquinando posibilidades para poner a los políticos de la región contra la pared y apretarles las tuercas (Mendoza, 2007, p. 177).

Los tres componentes antes mencionados se presentan en la situación que vive Gerardo, el tiempo que comparten y la cercanía que se genera entre los tres, como lo menciona la narración, hace que nazca una relación de amistad de la cual disfruta. Mariano y Candela, además de representar la lucha y la esperanza del pueblo, generan en Gerardo admiración por su labor. Ambos lo acogen a con el amor fraterno de la familia que él necesita en esta etapa de su vida. El hombre desorientado y lleno de dolor comienza a disfrutar de la compañía de los demás y se deja tocar por los propósitos 
y metas de los otros. En palabras de Längle, se permite ser tocado por las vivencias de los demás, y de esta manera se cumple la segunda motivación.

La tercera motivación, por su parte, plantea el permitirse ser uno mismo o el descubrimiento de sí y de la ética, pues al consolidar quién se puede ser y tener las ganas de vivir, el paso a seguir, como a continuación lo propone Längle (2007), es ser auténtico al aceptarse y proyectarse al mundo:

Yo soy yo, pero, ¿me siento inconfundible y único? ¿Siento que se me permite y alienta a ser como soy y a comportarme de la manera que lo hago? ¿Experimento el reconocimiento, respeto y estima por mi propio valor? Estas experiencias y sentimientos surgen como resultado de: consideración, justicia y aprecio (p. 32).

De acuerdo con los planteamientos de Längle, las experiencias y sentimientos son el fundamento de la autoestima, puesto que en la relación con los demás se comparte la esencia propia y del otro; en este sentido, se establece el reconocimiento de quien se es y, en esa diferencia, el valor de la propia vida y de ser único.

Para el caso de Gerardo, las experiencias y sentimientos que vivencia en la ciudad no contribuían al fortalecimiento de su autoestima: "Nada de lo que había hecho valía la pena. No tenía trabajo, mi madre había muerto en malas condiciones y mi padre estaba fuera de combate. ¿Por qué no irme? El sitio era lo de menos" (Mendoza, 2007, p. 38). En estas condiciones, el aprecio y la consideración eran ausentes, y por lo mismo la vida se le antojaba injusta; las pérdidas de sus seres queridos lo dejaban sin la posibilidad de experiencias, de encuentros con el otro desde la fraternidad y el afecto. Al no tener las experiencias y sentimientos que requiere, Gerardo asume que la desolación que siente está asociada al espacio de la ciudad, por lo que salir de allí representa la posibilidad de nuevos vínculos y afectos.

Esta motivación también es fundamental para Gerardo, ya que se descubre como la persona que no está en conformidad con lo que desea; al reconocerse y hacer un balance de quién es hoy, decide realizar un cambio contundente, no ser quien hasta ahora era: "Contemplé la posibilidad de cambiarme el nombre para que quedara claro que el actor Gerardo Montenegro no tenía nada que ver conmigo. Aunque alguna vez hubiera vivido en mi cuerpo" (Mendoza, 2007, p. 292).

En esta etapa, Gerardo se confronta consigo mismo y entiende la necesidad de desprenderse de quien ha sido hasta ahora; lejos de percibirse como alguien auténtico que se acepta, decide aniquilar al ser que ha constituido durante años. No reconocerse a sí mismo lo conduce a la elaboración de un nuevo Gerardo conforme a su deseo, y es el camino que 
emprende al iniciar su viaje de la ciudad a la selva: dejar al Gerardo citadino y construir al nuevo Gerardo en la selva, el cual se acepta como único y se proyecta ante el mundo.

La cuarta motivación que da cierre a las etapas que propone el Análisis Existencial en la búsqueda del sentido de vida consiste en pasar a la acción, hacer uso de las herramientas con las que se cuenta y ponerlas al servicio de sí mismo y de los demás:

La pregunta fundamental del continuo desarrollo y el cambio: Yo estoy aquí, pero, ¿para qué? ¿Con qué propósito? ¿En qué áreas o contextos más amplios me veo a mí mismo/a? ¿Para qué vivo? Esta dimensión de la existencia transforma nuestra contribución a la vida en un todo significativo (Längle, 2007, p. 32).

La pregunta que dota de sentido la existencia es ¿para qué vivo? Después de un largo proceso de reconocimiento de sí mismo y de experimentar las tres etapas anteriores, Gerardo comprende que su vida está en relación con los que ahora le rodean; para ello, ya hizo la elección del lugar para establecerse: el pequeño caserío de Noanamá en medio de la selva chocoana, donde los hombres que allí habitan y con los cuales compartirá hacen parte de su proyecto personal: la constitución del nuevo Gerardo, quien tiene como propósito otorgarle el sentido a la vida a través del servicio a los demás:

Farfullé para mis adentros: “¿Qué hay ahora más allá de mí mismo? Los otros. Me falta alcanzar ese último recuadro, estar entre los demás, gozar de su compañía, servirles, colaborar, saber que no estoy solo, que no soy la clave de nada, sino que hago parte de un todo que me complementa y me engrandece" (Mendoza, 2007, p. 287).

En esta etapa, Gerardo entiende que no se trata de preguntarse qué debo esperar de la vida, sino qué hago por mí y por los demás. Con respecto a su propio proceso de asignación de sentido de vida a su existencia, Gerardo dice con gran convicción:

Invertimos media vida en conocernos, en pensarnos, en entendernos por qué somos así y no de otro modo. [...] cuando por fin empezamos a hacernos responsables de cada concepto y de cada emoción que sentimos y que generamos, es cuando de verdad estamos listos para desplegar nuestra fuerza en el mundo y transformarlo de buena manera. ¿Qué hay más allá de nosotros mismos? Los otros, que están ahí esperándonos con los brazos abiertos (Mendoza, 2007, p. 286).

El sentido existencial de Gerardo llega a la plenitud cuando asume que es el servir a los otros, reconociéndose a sí mismo como único e importante, lo que le permitirá trascender.

El Sentido Existencial, definido por Längle como "la opción más valiosa ante cada situación", refiere a un "hacer", a una acción, a un salir desde sí, trascender de sí para ir al mundo que requiere de sí y donde se percibe necesitado. Sólo ahí se despliega el Ser, la Persona (Croquevielle, 2009, p. 31). 
El planteamiento de Längle (2009) sobre el Sentido Existencial enfatiza en "trascender de sí para ir al mundo que requiere de sí" (p. 31); evidentemente, la expresión de la cita no es plenamente literal; sin embargo, para el caso de Gerardo aplica el hecho de que el mundo que lo requiere es un espacio físico particular: la selva chocoana, y particularmente el leprocomio de Noanamá. Este espacio representaba todo lo que Gerardo aún tenía por entender de su existencia: la enfermedad como oportunidad de encuentro, la fraternidad, la construcción de comunidad, el disfrute de la vida sencilla y el servicio a los demás. La ciudad ya le había quitado y ofrecido lo que conocía y lo que hasta ese momento era él; ahora su sentido de vida, su renacer estaba en función de los demás en el espacio en el cual se sentía necesitado, valorado y con la firme convicción de que su decisión de establecerse en Noanamá representaba el encuentro con su búsqueda, la del sentido de su vida.

El viaje que inicia Gerardo es la búsqueda de sí mismo, propósito que logra encontrar a medida que avanza. Cada una de las motivaciones propuestas por el Análisis Existencial son etapas de la vivencia que hacen posible su realización personal, pese a las exigencias y dificultades que halla en su entorno inicial y posteriormente en su trasegar. El camino que emprende hacia el exterior se convierte también en un camino al interior de sí mismo, de manera tal que logra conducirse al lugar más distante y disímil de la ciudad: la selva. De esta misma manera, llega al final del camino interno, al encontrar el sentido de su existencia y el lugar en el mundo en el cual establecerse en concordancia con su proyecto de vida.

La coherencia interna o el "consentimiento interior" que menciona Längle (2007, p. 30) corresponde con la meta a la cual llega Gerardo; todas sus acciones con respecto al propósito de dejar de ser él mismo se cumplen, en tanto el giro que da a su existencia obedece a un plan que se va configurando y ajustando a medida que avanza en su viaje. La plenitud de la cual se enorgullece la encuentra al tomar distancia de todo lo que constituye su espacio de ciudad, y el empezar de nuevo incluye ese nuevo lugar en el cual reconoce a otras personas con las cuales vincularse a propósitos que progresivamente llegan a ser suyos, al empezar a configurar la comunidad que desea y a la cual ya pertenece.

Durante todo su viaje, Gerardo Montenegro llevó a cabo las cuatro fases o motivaciones que plantea Längle en el Análisis Existencial para la búsqueda del sentido de la existencia, siendo la última el planteamiento que 
en su momento da inicio a la Logoterapia de Frankl, y que se constituye en la antesala del sentido de vida propuesto por él y posteriormente desarrollado por Längle.

\section{A modo de conclusión}

Nos hemos ocupado de una coyuntura crucial de la novela Los hombres invisibles, en la que el protagonista apunta a la necesidad de otorgarle sentido a la existencia mediante el desplazamiento de la ciudad a la selva. Para ello se propone la búsqueda de la comunidad de los hombres invisibles, y aunque inicialmente se lo planteó como una excusa para salir de la ciudad, a medida que avanza en su recorrido se descubre que su verdadera pretensión no es otra que la búsqueda de sí mismo, y de esta manera encontrar un lugar en el mundo para establecerse y darle un sentido a su vida. Gerardo inicia así un recorrido que si bien es incierto en su primera parte, se consolida paulatinamente hasta alcanzar su objetivo.

Son pues las decisiones de Gerardo, y los aprendizajes que ellas generan, las que van dándole sentido a su vida. Es de esta forma que el protagonista logra trascender el dolor de las pérdidas y encontrar un lugar en el mundo para llevar a cabo su proyecto personal; esa tarea exige un nuevo comienzo enmarcado por el eco de los versos de Kavafis que Gerardo trae a su memoria:

La ciudad es una jaula.

No hay otro lugar, siempre el mismo

Puerto terreno, y no hay barco

Que te arranque a ti mismo. ¡Ah! ¿No comprendes

Que al arruinar tu vida entera

En este sitio, la has malogrado

En cualquier parte del mundo?

El poema no se cumpliría. No terminaría prisionero en la misma celda de cemento ni había arruinado mi vida en todos los lugares del planeta. Mi destino estaba muy lejos de la ciudad, en medio de la selva, entre los brazos leprosos de Tehura (Mendoza, 2007, pp. 295-296).

Tal como lo plantean Frankl y Längle, emprender un proyecto que le genere gozo y sentido a la existencia, en el caso del protagonista de Mendoza, es lo que descubre al decidir emprender su viaje, que es en sí mismo un recorrido interior y que posteriormente se materializa al establecerse en Noanamá.

El contrastar los espacios específicos tratados en la novela hizo posible que se reconocieran como contrarios irreconciliables, siendo la ciudad representada como un ente malvado que ataca y daña a quienes la habitan, y 
la selva como un lugar sagrado y ancestral que, al no ser tocado por el hombre de ciudad, conserva una especie de pureza.

Es por ello que el sentido de vida en relación con lo urbano en la novela se manifiesta como un desafío para el ser humano que se enfrenta a las dinámicas atomizantes y deshumanizadas que propone la ciudad. El ser citadino se cuestiona y hastía a tal punto que busca reconocerse en otros espacios y con otros seres. La necesidad de salir de los convencionalismos y configurar una existencia más profunda, es lo que se disputa en dicha relación.

Finalmente, la búsqueda de Gerardo Montenegro demuestra que encontrar sentido a la existencia es un imperativo en el ser humano, así como encontrar un lugar en el mundo dónde establecerse y llevar a cabo su proyecto de vida es determinante.

El artículo abre la puerta a posteriores desarrollos que busquen rastrear esto mismo en otras novelas, o apoyarse en otras escuelas psicoterapéuticas, para explorar más la relación psicoterapia-narración.

\section{Referencias bibliográficas}

1. Chávez, M. I. (2009). Alfred Adler y Viktor Frankl: El discurso acerca del sentido de la vida. Avances en psicologia 17 (1), pp. 35-58.

2. Croquevielle, M. (2009). Análisis existencial: sus bases epistemológicas y filosóficas. Castalia: Revista de Psicología de la Academia 15, pp. 23-34.

3. Frankl, V. (1967). Logoterapia y existencialismo. Barcelona: Herder.

4. Frankl, V. (1991). El hombre en busca de sentido. Barcelona: Herder.

5. Längle, A. (1998). Las cuatro condiciones fundamentales para una existencia plena. Viena: E Suess Gasse. Disponible en https://bit.ly/2qDdG7O [21.12.2017].

6. Längle, A. (2007). Análisis Existencial: búsqueda de sentido y afirmación de la vida. Revista de Psicología UCA 3 (5), pp. 5-24.

7. Längle, A. (2009). Las Motivaciones Personales Fundamentales. Revista de Psicología UCA 5 (10), pp. 7-24.

8. Mendoza, M. (2007). Los hombres invisibles. Bogotá: Planeta.

9. Pareja Herrera, L. G. (1998). Viktor Frankl: comunicación y resistencia. Coyoacán: Fontamara. 